\title{
Wie alles herausgekommen ist ...
}

\section{Markus Gnädinger}

Facharzt für Innere Medizin, Qualitätszirkel Oberthurgau
Korrespondenz:

Dr. med. Markus Gnädinger

Birkenweg 8

CH-9323 Steinach

Tel. 0714460464

markus.gnaedinger[at]hin.ch
Im Nachgang zu unserer Selbstanzeige [1] und deren Echo in der Fach- [2-4] und Laienpresse [5, 6] hat die Leserschaft der Schweizerischen Ärztezeitung ein Recht, zu erfahren, wie sich die Geschichte mit unserem Strafverfahren beim Heilmittelinstitut Swissmedic entwickelt hat. Zudem versuche ich, eine kleine Auslegeordnung zur «kleinen» klinischen Forschung in unserem Land zu erstellen.

Als Hauptverantwortlicher für den illegalen hausärztlichen Selbstversuch zur Nahrungsabhängigkeit der intestinalen Vitamin-D-Absorption [7] wurde ich von Swissmedic einvernommen. Nach diversen Korrespondenzen wurden meine 13 Kollegen und ich wegen Verstosses gegen das Heilmittelgesetz mit zirka je 1000 CHF Busse (Studienleiter $4000 \mathrm{CHF}$ ) und $240 \mathrm{CHF}$ Verfahrenskosten, total also gut 20000 CHF gebüsst. Wegen Aussichtslosigkeit haben wir keine Einsprache erhoben; diese Bussen sind nun rechtskräftig.

Wir glauben, dass die von uns angestossenen Diskussionen im öffentlichen Interesse waren. Es besteht ein ethisches Dilemma: Wie schwer wiegt der Umstand, dass eine forschungsfreundliche Regelung durch Industrie oder Wissenschaftler missbraucht werden könnte? Und wie schwer das Unwissen, das durch eine (Über-?)Reglementierung und somit die Verhinderung notwendiger Studien geschaffen wird? Ist es schlimmer, wenn zehn Personen in einer Studie geschädigt werden (und dies wissen) oder hundert Patienten durch eine insuffizient erforschte Off-label-Behandlung beeinträchtigt werden (und dabei natürlich nicht wissen, dass man

\section{Etude sur la vitamine D: des généralistes jouent les cobayes}

14 généralistes ayant testé de la vitamine $D$ sur euxmêmes se sont vus infligés une amende/des frais de procédure à hauteur de 20460 francs. Dès 2014, grâce à de nouvelles bases légales, de nouvelles sources de financement et des cours de bonne pratique clinique destinés aux généralistes et aux pédiatres, il sera possible de combler le retard en matière de recherche dans ce domaine.

denen einerseits das gegenseitige Verständnis gefördert und andererseits auf gewisse Bestimmungen des Verordnungsrechtes zum neuen, ab dem 1.1.2014 gültigen Humanforschungsgesetz (HFG) Einfluss genommen werden konnte. Es wird nun auch ein scharfes Auge auf die Bewilligungspraxis nach neuem Recht brauchen; insbesondere wird die Anwendung von KlinV Art. 19, Abs. 4 (Herabstufung eines Versuchs von der strengeren Kategorie B zu A) kritisch zu beobachten und ggf. zu monieren sein.

Wäre unsere Vitamin-D-Studie nach neuem Recht bewilligt worden? Es ist meines Erachtens klar, dass der Gesetzgeber weder beim alten Heilmittelgesetz noch beim neuen HFG eine Gruppe von Hausärzten vor Augen hatte, die einen Selbstversuch mit einem frei verkäuflichen Vitamin (Kategorie D)

\section{Es ist klar geworden, dass die rechtlich-ethischen und administrativen Hürden für Medikamentenforschung am Menschen in unserem Land sehr hoch sind.}

diese Therapie längst hätte genauer erforschen können)? Das Nicht-Vorhandensein von Information hat leider oft keinen grossen Nachrichtenwert!

Was haben wir mit unserer Aktion erreicht? Es ist dem interessierten Publikum klar geworden, dass die rechtlich-ethischen und administrativen Hürden für Medikamentenforschung am Menschen in unserem Land sehr hoch sind. Seitens der hausarztmedizinischen Institute haben mit den zuständigen Beamten des BAG verschiedene Gespräche stattgefunden, bei durchführt. Auch im neuen HFG wäre der Versuch aber nicht bewilligt worden, da sich die Studienärzte nicht über den notwendigen Sachverstand ausweisen konnten (sprich: absolvierter Kurs über Good Clinical Practice, GCP).

Das neue HFG versucht, einen Interessenausgleich zwischen den Bedürfnissen der Forschung und dem Schutz der Versuchspersonen zu schaffen. Neben der konzisen Festschreibung des geltenden Rechts und dem Stopfen bisheriger Lücken bringt 


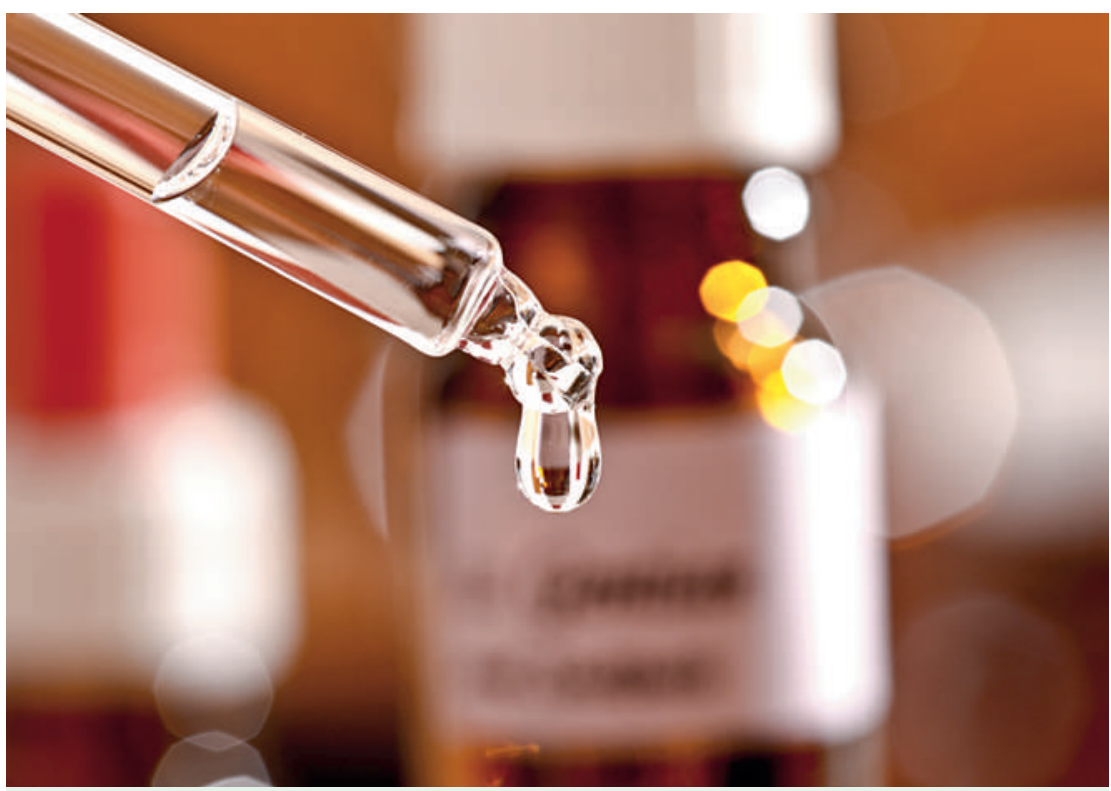

Der Tropfen des Anstosses: ein nicht genehmigter Selbstversuch zur Bioverfügbarkeit von Vitamin-D-Supplementen, durchgeführt mit öligen Tropfen. und ein nationales Forschungsprogramm ist in Aussicht [8].

Bei der Forschung in der Hausarztmedizin geht es zwar nicht nur, aber auch um den Einsatz von Medikamenten. Auch diesbezüglich sollte es besser werden: Für Haus- und Kinderärzte werden nächstes Jahr GCP-Kurse angeboten, was die Basis dafür schafft, dass überhaupt klinische Studien stattfinden können. Da die Forschung, zumal an nicht mehr patentgeschützten Medikamenten, jedoch weiterhin defizitär ist [9], sollte aber eine nachhaltige Finanzierung derselben angestrebt werden, z. B. durch Äufnen von 1\% des Exfactory-Preises aller patentfreier Arzneien in einen Studienfonds, damit die aktuellen Bemühungen kein Strohfeuer bleiben. Aber nicht nur das fehlende Geld ist ein Problem, sondern auch der Umstand, dass Fachinformationen nicht korrekt nachgeführt werden und dass sich niemand mehr um das Nebenwirkungsprofil altbekannter Produkte kümmert. Hier sind Politik und Verwaltung gefordert, um die notwendigen Anreize bei den Herstellern zu schaffen.

\section{Literatur}

1 Gnädinger M. Die Kraft, die Böses will, doch Gutes schafft. Schweiz Med Forum. 2012;12(43):841-3.

2 Gnädinger M, Bossert F, Eichmann F, Haug B, Krüsi M, Nadig M et al. Ceci n'est pas une étude. Schweiz Ärztezeitung. 2013; 94(7): 261-3.

3 Altorfer R. Wenn Hausärzte forschen oder von Notifikationen, Ethikkommissionen, GCP und HFG. Ars Medici. 2013;4:188-9.

4 Gnädinger M. Einseitige Reglementation. Schweiz Ärztezeitung. 2013;94(41):1541

5 Straumann F. Mit Vitamin D gegen die Bürokratie. Tagesanzeiger 2013. Mittwoch, 13.3.2013. S. 34.

6 Andresh J. Gegen jede Regel. Frankfurter Allgemeine Sonntagszeitung vom 12.5.2013. S. 52.

7 Gnädinger M, Bossert F, Eichmann F, et al. Oral vitamin D - Is it Necessary to be taken with Meals Containing Fat? WebmedCentral Endocrinology. 2013; 4(2):WMC004023.

8 Rosemann T, Bischoff T, Schaufelberger M, Sommer J, Tschudi P. Forschung in der Hausarztmedizin. Schweiz Ärztezeitung. 2013;94(22):823-4.

9 Gysling E. Die Basis unserer Pharmakotherapie ist defizitär. pharma-kritik. 2012;34(10):37-9. 